\title{
Controle de consumo de água baseado em reconhecimento facial
}

\author{
Pedro E. Santos ${ }^{1}$, Roubert Edgar S. Silva ${ }^{1}$, \\ Dalmy F. Carvalho Jr ${ }^{1}$, Ilo A. S. Rivero ${ }^{1}$, Raquel A. F. Mini ${ }^{2}$ \\ ${ }^{1}$ Faculdade de Engenharia - Universidade de Itaúna (UI) \\ Rodovia MG 431 km 45, s/n - Campus Verde, Itaúna - MG, 35.680-142 - Brasil \\ ${ }^{2}$ Departamento de Informática - Pontifícia Universidade Católica de Minas Gerais \\ Rua Walter Ianni, 255 - São Gabriel - Belo Horizonte/MG - Brasil
}

\begin{abstract}
The ubiquitous computing aims to be invisible. From that, we can identify some individuals in an environment, allowing context aware and personalized services. Technologies like that can help water demand control, avoiding domestic and industrial water waste. This paper proposes a home water consumption control, based on facial recognition and wireless sensor networks (WSN) controlled by a mobile device. The water flow can be controlled and monitored after user's facial recognition. An experiment was developed to monitor and control water flow using real time face recognition. The results show that the proposed system can save up to $50 \%$ water consumption.
\end{abstract}

Resumo. A computação ubíqua possui como objetivo principal a presença uniforme e imperceptível na vida cotidiana do ser humano. A partir disso, é possível, além de outros recursos, identificar indivíduos em um ambiente, permitindo serviços personalizados e sensíveis ao contexto. Tecnologias como essa podem auxiliar o controle da demanda de recursos hídricos, afetados diretamente pelo desperdício contínuo domiciliar e industrial. Este trabalho propõe uma solução para o controle do consumo de água residencial, baseado em reconhecimento facial. Utilizando uma rede de sensores sem fio (RSSF) interligada a um dispositivo móvel, um usuário é identificado por meio da face para regular a vazão e monitorar a quantidade de água utilizada por ele. Desenvolveu-se um protótipo para monitorar e controlar esse consumo, que é ajustado em tempo real de acordo com a face do usuário identificado. Os resultados mostram que é possível economizar até $50 \%$ do consumo de água com a utilização do sistema proposto.

\section{Introdução}

A utilização dos recursos hídricos cresceu mais que o dobro do crescimento populacional nas últimas décadas [WWAP 2015]. Esse crescimento se relaciona diretamente a práticas pouco estruturadas de consumo no meio agrícola, industrial e urbano. Consequentemente, há um aumento do estresse hídrico em regiões onde a demanda por água é maior do que a sua disponibilidade e capacidade de renovação [FAO 2011].

O desperdício de água é vivenciado em todo o Brasil. Segundo a Organização das Nações Unidas (ONU), cada indivíduo necessita de aproximadamente 110 litros de água por dia para atender suas necessidades de consumo e higiene [Morais 2015]. De 
acordo com o relatório fornecido pela Secretaria Nacional de Saneamento Ambiental [SNSA 2014], o país possui uma média de consumo de 166, 3 litros de água por habitante ao dia, podendo chegar a 253,5 litros em regiões onde a utilização do recurso não é devidamente controlada.

O objetivo deste trabalho é apresentar um sistema de monitoramento e controle do fluxo de água orientado à identificação facial de indivíduos em tempo real, desenvolvido com a utilização dos conceitos de visão computacional, microcontroladores e redes de sensores sem fio (RSSF). Com a integração do reconhecimento facial em um dispositivo móvel, interligado a uma central controladora de uma RSSF, é possível automatizar o controle da utilização da água dentro de uma residência pelos indivíduos residentes. Em relação à automação domiciliar, [Bolzani 2004] coloca que ela deve ser utilizada para ajudar o usuário a cumprir suas tarefas diárias, mas não a pensar por ele. As informações devem estar precisamente dispostas no tempo e no lugar necessários auxiliando na tomada de decisões e, através de aprendizado e adaptação, fornecer sugestões para o gerenciamento dos sistemas.

\section{Reconhecimento facial}

$\mathrm{Na}$ linguagem da Teoria da Informação, a extração de informações relevantes em uma imagem de face, codificação eficiente dos dados e uma precisa comparação de uma face com outros modelos codificados em uma base de dados são pontos importantes para que a técnica de reconhecimento seja praticável. O corrente projeto utiliza como algoritmo de reconhecimento facial a técnica de extração de texturas LBP (Local Binary Patterns).Nesse algoritmo, as faces podem ser vistas como uma composição de micropadrões que são bem descritos por esta técnica [Ojala et al. 1996].

$\mathrm{O}$ algoritmo LBP se baseia na intensidade dos pixels de uma imagem. A intensidade $v_{c}$ de cada pixel de uma imagem é substituída por um vetor binário $b$, determinado pela Equação 1, que mostra a comparação entre a intensidade do pixel e as intensidades dos pixeis vizinhos [do Amaral et al. ].

$$
b_{i j}=\left\{\begin{array}{c}
0, v_{i j}<v_{c} \\
1, v_{i j} \geq v_{c}
\end{array},\right.
$$

$\mathrm{Na}$ Equação 1, $v_{i j}$ corresponde ao valor de um pixel na posição $(i, j)$, sendo $1 \leqslant i, j \leqslant N$. $N$ corresponde ao tamanho da vizinhança, também chamada de máscara. Os valores obtidos para cada vizinho são, então, concatenados, gerando um número binário. Esse número obtido é convertido para sua base decimal substituindo o valor central $v_{c}$.

Os descritores de textura do LBP são obtidos isoladamente de regiões da face, comumente chamadas de malhas regulares. Após essa extração, são calculados os histogramas de intensidade dos pixels da imagem, resultando em valores que são concatenados e um único vetor de histogramas, facilitando a comparação e análise de amostras.

O processo chave do algoritmo se encontra nas classificações das regiões da imagem. No processo de classificação utilizam-se, basicamente, métricas que permitam explorar a importância de certas características faciais em relação a outras [Zhao et al. 2003]. Portanto, para cada região $j$ da malha de histogramas, é possível 
atribuir-se pesos específicos. Um exemplo de definição de pesos em regiões pode ser visto na Equação 2 de distância Qui-Quadrado [Ahonen et al. 2006].

$$
X_{w}^{2}(x, y)=\sum_{i, j} w_{j} \frac{\left(x_{i, j}-y_{i, j}\right)^{2}}{x_{i, j}+y_{i, j}}
$$

Na Equação 2, $x$ e $y$ são matrizes de histogramas a serem comparadas, $i$ como um elemento pertencente a $j$-ésima região e $w_{j}$ representando o peso pré-definido para a região $j$.

O algoritmo foi escolhido por haver melhores documentações a seu respeito, além de estar disponível em código aberto por meio da biblioteca OpenCV de visão computacional. O LBP vem sendo estudado bastante nos últimos anos, como pode ser visto em [Chan et al. 2007] e [Zhang et al. 2010]. O fato do algoritmo se apresentar de forma relativamente simples quanto à sua implementação também contribui para sua utilização.

\section{Redes de sensores sem fio}

Uma rede de sensores sem fio (RSSF) é definida por uma infraestrutura composta de sensoriamento, processamento e intercomunicação, realizada por um número considerável de dispositivos sem fio (nós sensores) situados em uma região de interesse, que pode ser utilizada para instrumentar, observar e reagir a eventos e fenômenos em ambientes específicos [Sohraby et al. 2007]. Uma RSSF foi utilizada em [Suprotim Sinha Majumdar and Alamelu 2015] para monitorar mudanças climáticas em plantações, permitindo um melhor controle do consumo de recursos, como água e energia.

As RSSF podem ser utilizadas para monitoração residencial, foco deste trabalho. [Rizzoli 2015] discute a importância no desenvolvimento de soluções que possam reduzir e controlar o consumo de água, e, principalmente, a importância de se conhecer o comportamento do indivíduo ao utilizar água. Este trabalho apresenta uma solução para monitorar o consumo individual, baseado na identificação visual de cada usuário de uma residência.

O nó sensor é desenvolvido com base na plataforma de prototipagem eletrônica Arduino, utilizando o microcontrolador ATMega 328 AVR 8-bit, sob um conjunto de instruções RISC [Atmel 2014]. Escolheu-se a plataforma com intuito de agilizar o processo de prototipagem dos nós sensores. A comunicação entre os nós da RSSF é realizada por meio do padrão ZigBee implementado sobre o padrão IEEE 802.15.4.

\subsection{Sensoriamento e Atuação}

Para as atividades de monitoramento do fluxo de água são utilizados sensores de fluxo de efeito hall. Os sensores utilizados possuem $\frac{1}{2}$ polegada de diâmetro e trabalham tensionados por $5 \mathrm{~V}$. Cada sensor possui apenas três fios para alimentação $(V C C)$, aterramento $(G N D)$ e transmissão dos pulsos gerados durante a utilização de um dispositivo hídrico. O cabo condutor de pulsos deve ser ligado a uma entrada digital do Arduino, para que os pulsos gerados sejam recebidos e contabilizados. Para isso, são utilizadas as funções de interrupção do Arduino (PCINT-Pin Change Interrupt), disponíveis nos pinos 2 e 3. 
O fluxo de água é controlado com a utilização de um servo motor atuando sobre um registro esférico de $\frac{1}{2}$ polegada e permite ajustes quantitativos de seu volume utilizado durante determinado instante de tempo. O protótipo elaborado permite ajustes quantitativos do fluxo de água em litros por minutos. A vazão em cada nó sensor é definida pelo usuário com a utilização da central de controle desenvolvida para tratamento e armazenamento dos dados oriundos do monitoramento, além do controle dos nós sensores.

\subsection{Aplicação móvel de reconhecimento}

O processo de detecção facial ocorre em um programa móvel, podendo ser utilizado em qualquer lugar, desde que haja luminosidade o suficiente para que um rosto seja detectado ou reconhecido. A aplicação de detecção facial de um indivíduo é uma das etapas do sistema completo. Ao haver a detecção de um membro domiciliar, suas atividades envolvendo recursos hídricos são enviadas a uma central de controle de dados, como descrito melhor na Seção 4.3. Foi desenvolvido um aplicativo para dispositivos Android. Ao iniciar a aplicação, é possível detectar uma face e realizar o cadastramento do indivíduo, reconhecê-lo após o cadastramento e visualizar os cadastros realizados.

\subsection{Detecção Facial}

O sistema de detecção e reconhecimento facial é composto pelas seguintes etapas: aquisição da imagem; pré-processamento da imagem adquirida; extração das características da imagem e classificação e verificação de reconhecimento da imagem. O diagrama da arquitetura do sistema de detecção e reconhecimento facial pode ser vista na Figura 1.

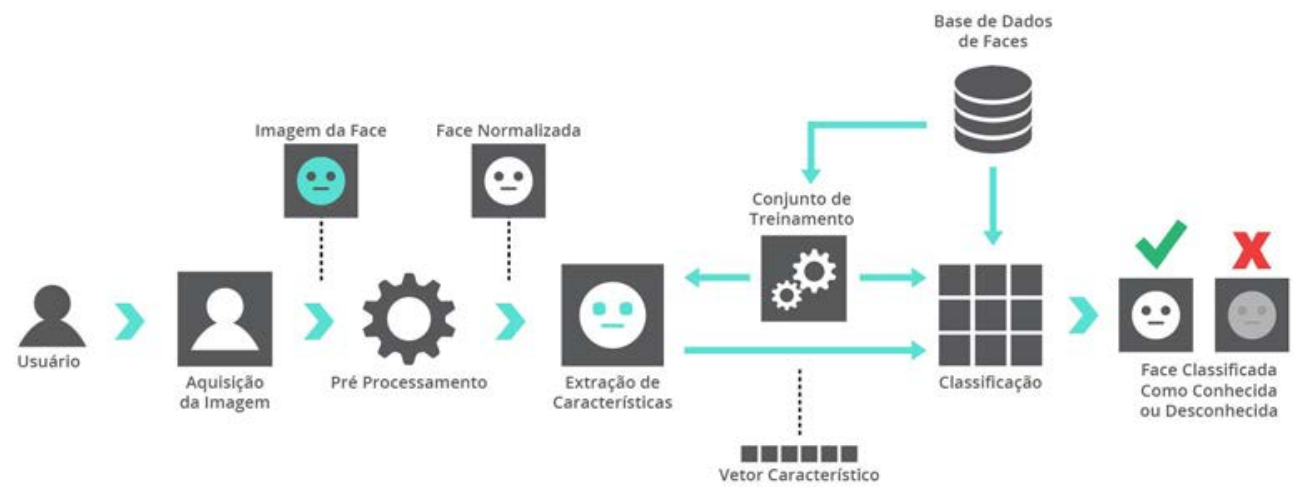

Figura 1. Arquitetura do sistema de reconhecimento de face

O processo do sistema inicia-se pela arquitetura do reconhecimento facial, na aquisição das imagens a serem analisadas. Uma imagem é capturada em tempo real, por meio da câmera do dispositivo móvel. Após a captura, a imagem é processada para servir de entrada para o reconhecimento e classificação final das faces. O resultado final consiste em uma imagem recortada e em escala de cinza, contendo apenas a face do indivíduo, para um melhor funcionamento dos métodos subsequentes da arquitetura. A captura e extração de imagens faciais para persistência são realizadas utilizando a biblioteca OpenCV (Open Source Computer Vision Library) de processamentos gráficos.

OpenCV [Bradski and Kaehler 2008] é uma biblioteca voltada à visão computacional utilizada neste trabalho. O OpenCV possui módulos de processamento de imagens 
e vídeo, estrutura de dados, álgebra linear, interface gráfica do usuário (GUI), controle de mouse e teclado, além de mais de 2500 algoritmos, muitos dos quais são considerados estado da arte, tais como segmentação, detecção de faces (método Viola-Jones [Viola and Jones 2004]), aprendizado de máquinas, filtragem de imagens, rastreamento de movimento, entre outros métodos [Diniz et al. 2013].

\subsection{Interface de reconhecimento de usuários}

O usuário interage com o sistema apenas utilizando um dispositivo móvel, capturando informações faciais de indivíduos e posicionando o aparelho móvel em locais de fácil captura de rostos. A Figura 2 ilustra a interface principal da plataforma móvel, contendo botões de captura de faces e disparo da rotina de reconhecimento facial.

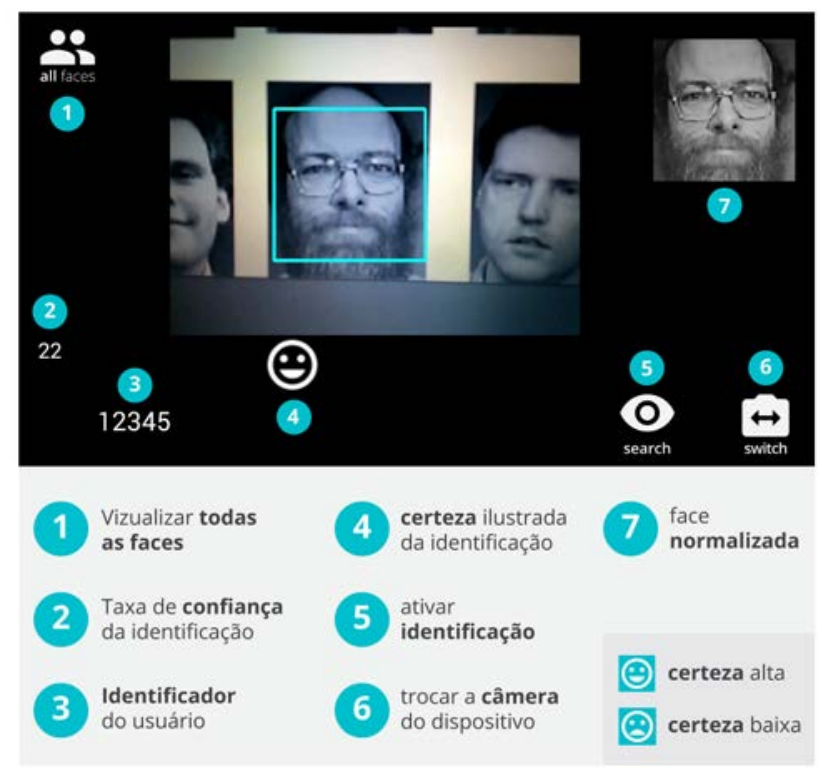

Figura 2. Interface do protótipo de reconhecimento facial móvel

As características da imagem obtidas pelo método anterior são, então, extraídas e comparadas com as imagens do conjunto de treinamento. Desse modo, a partir da comparação, é possível saber se uma face existe ou não na base de dados.

\section{Monitoramento e controle do fluxo de água}

\subsection{Monitoramento}

Os módulos da RSSF são compostos por um sensor de fluxo, um controlador de fluxo e um rádio transceptor. Durante o monitoramento do fluxo de água tem-se a execução das seguintes tarefas: coleta de dados referentes ao fluxo de água coletados pelos sensores de fluxo; os dados são transmitidos pela rede de sensores à uma central de controle; os dados são tratados e armazenados na central de controle e, por fim, os dados são apresentados ao usuário sob demanda.

O sensor de fluxo utilizado na atividade de monitoramento foi programado para fornecer a quantidade total de pulsos gerados durante a utilização de um dispositivo 
hídrico. Ao tornar a geração de pulsos nula, ou seja, quando não há mais fluxo corrente no sensor, o número de pulsos contabilizado é enviado à central de controle.

O tratamento dos dados recebidos na central de controle é realizado com a divisão do número total de pulsos gerados durante uma atividade doméstica pela quantidade de pulsos por litro de água. A quantidade de pulsos por litro de água é obtida por meio da calibragem do sensor de fluxo, realizada com a contagem de pulsos durante o preenchimento de um recipiente até a marca de 1 litro. Após uma sequência de dez experimentos, obteve-se uma média de 378, 9 pulsos por litro de água, com um coeficiente de variação menor que $3 \%$, dentro dos limites definidos pelo fabricante.

A mensagem recebida na central de controle é tratada com a divisão do valor recebido (número total de pulsos gerados) por 380, o que define a quantidade de litros de água utilizados. Após o processo de tratamento, os dados são armazenados com a inclusão do nome do nó que originou a mensagem, seu endereço de 64bits e a data e hora do registro.

\subsection{Controle do fluxo de água}

O controle de fluxo é realizado com a utilização de um controlador de fluxo que atua sobre a vazão de água em um dispositivo hídrico. Essa vazão é determinada na central de controle e definida no destino após a transmissão da mensagem do nó coordenador ao nó sensor. As etapas do controle de fluxo são:

- Definir o fluxo máximo de água em um nó sensor utilizando a central de controle;

- Transmitir a mensagem contendo o valor do fluxo definido ao nó destino;

- Analisar o fluxo corrente no sensor e realizar ajustes no fluxo de água em tempo de utilização.

O algoritmo desenvolvido para o controle de fluxo é integrado ao algoritmo de monitoramento. Como apresentado na sessão 4.1, apenas o número total de pulsos gerados durante toda a atividade doméstica foi utilizado, com o incremento progressivo a cada segundo. A necessidade de um comparador para que fosse medido o volume de água corrente no sensor é suprida com a integração de um contador de pulsos. O contador é zerado a cada segundo e fornece a vazão em litros de água por minuto utilizando a equação, disponibilizada pelo fabricante do sensor de fluxo de água, apresentada na Equação 3.

$$
\text { litros } / \text { minuto }=\frac{P}{7.5}
$$

Na Equação 3, $P$ é o número de pulsos gerados durante o delay de um segundo dividido por 7,5 , constante definida pelo fabricante para o sensor de fluxo de água de $\frac{1}{2}$ polegada.

Com a base de comparação definida, é possível realizar comparações entre o volume de água que está sendo utilizado e o valor definido na central de controle, permitindo ajustes do fluxo de água em tempo de utilização de determinado dispositivo hidráulico por um indivíduo.

\subsection{Integração intersistêmica}

O processo final de elaboração do sistema proposto consiste em integrar o sistema de reconhecimento facial ao sistema de monitoramento e controle do fluxo de água. A junção 
dos dois sistemas ocorre na etapa de tratamento dos dados na central de controle. Durante a etapa de processamento, que precede a etapa de armazenagem dos dados, o usuário cadastrado que teve sua face detectada no sistema, é vinculado a todos os registros originados no local onde o reconhecimento foi realizado. A partir do momento em que o usuário finaliza seu uso em um dispositivo hídrico, sendo ele uma torneira ou chuveiro, os dados obtidos a partir do fluxo são enviados à central de controle para a exibição dos gastos, onde é possível verificar se há desperdício no local monitorado.

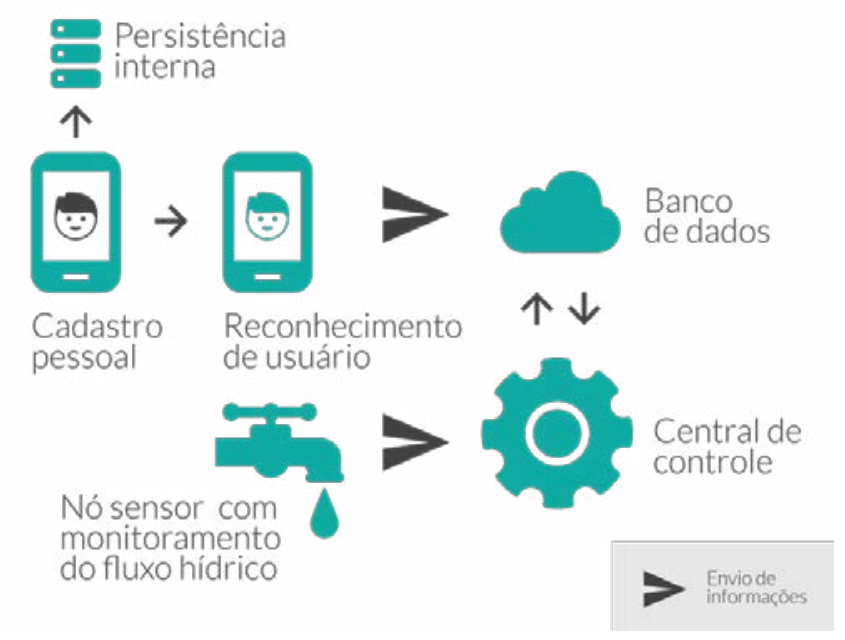

Figura 3. Processo de reconhecimento de indivíduos e monitoramento do fluxo hídrico

\section{Resultados e validações}

\subsection{Resultados do Monitoramento}

O sistema de monitoramento do fluxo de água apresentou resultados satisfatórios durante sua utilização, visto que a obtenção, transmissão, tratamento e armazenamento dos dados foram realizadas com sucesso. A obtenção dos dados foi realizada em uma residência com 5 moradores. Instalou-se o nó sensor em uma torneira localizada na cozinha da residência, com o sistema em funcionamento por aproximadamente 4 horas. Ao término dos experimentos de monitoramento, foi possível obter os resultados apresentados na figura 4

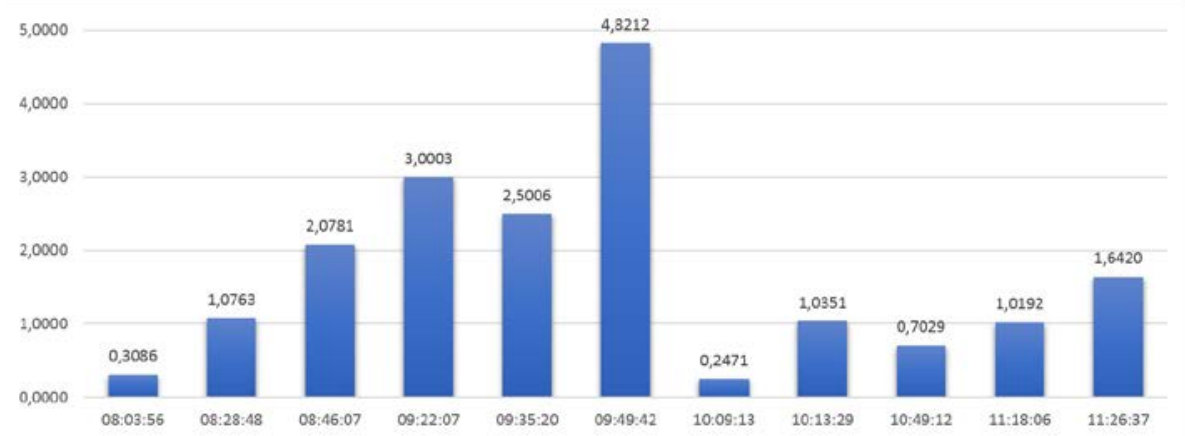

Figura 4. Resultados do monitoramento em um intervalo temporal 
O gráfico apresentado na figura 4 mostra o monitoramento do fluxo de água e apresenta o eixo $Y$ como sendo a quantidade d'água utilizados e o eixo $X$ representando os horários das medições. O sistema trabalha com instantes de utilização, sendo possível visualizar a hora do recebimento da mensagem contendo a quantidade de litros gastos durante determinado intervalo de tempo.

\subsection{Resultados do controle de fluxo}

Durante o controle de fluxo, nenhum dado referente ao volume de água foi transferido à central de monitoramento, visto que toda persistência é voltada ao monitoramento. Os resultados foram obtidos através de uma aplicação desenvolvida em $\mathrm{C}++$. Os dados apresentados foram registrados com a utilização do monitoramento serial por meio do método Serial.print(). A cada segundo, o valor obtido pelo cálculo de litros por minuto é impresso no monitor Serial. O histórico de medição é registrado no terminal de monitoramento, permitindo a extração dos resultados, sendo desnecessária a persistência de tais dados no banco de dados.

Foram realizados três experimentos de redução do fluxo de água. O primeiro experimento não conta com qualquer controle do fluxo, fornecendo a base de cálculo para a eficiência das reduções realizadas na sequência. Durante os experimentos, sem qualquer limitação de fluxo, foram obtidas 26 amostras. As quatro primeiras e quatro últimas amostras de dados foram descartadas, visto que as quatro primeiras registram a abertura da torneira e as quatro ultimas registram seu fechamento. A média obtida durante os experimentos foi de 12 litros/minuto com a torneira totalmente aberta.

O segundo experimento foi realizado com a limitação do fluxo de água em 9 litros/minuto. Os resultados se mostram satisfatórios, apesar da baixa eficiência do algoritmo em tratar a redução de fluxo, visto que, se o fluxo é menor do que o valor determinado, entende-se que o controlador deve aumentar sua vazão até atingir seu ponto máximo (definido como 105 graus). O controlador de fluxo aumenta em 5 graus a abertura da válvula de fluxo de água progressivamente, até atingir o momento em que o fluxo de água se encontra próximo ou é igual ao valor delimitado. O controlador de fluxo é, então, desabilitado com a utilização do comando detatch(PIN) no pino 11. Sua ativação é somente realizada novamente quando uma mensagem para limitação de fluxo é recebida.

O terceiro experimento de controle de fluxo foi realizado com o fluxo de água limitado a 6 litros/minuto. As diferenças no consumo de água dos três experimentos são apresentados na figura 5, onde é possível observar o problema do algoritmo de redução do fluxo. Na delimitação do fluxo em 9 litros por minuto, é possível observar que o fluxo ultrapassou o limite e só depois foi reduzido. Durante a redução, o fluxo chegou a ser menor do que o valor máximo e estabilizado no segundo seguinte, quando a diferença entre o valor delimitado (9 litros/minuto) e o valor corrente no sensor era menor ou igual a $\frac{1}{2}$. O ocorre quando o valor máximo definido para o fluxo de água foi de 6 litros/minuto.

\section{Considerações Finais}

\subsection{Conclusões}

O objetivo principal da prototipagem de reconhecimento de indivíduos foi alcançado. O protótipo realiza o reconhecimento facial e armazena seus dados, para posterior uso domiciliar e monitoramento individual dos recursos hídricos. O usuário é pré-cadastrado 


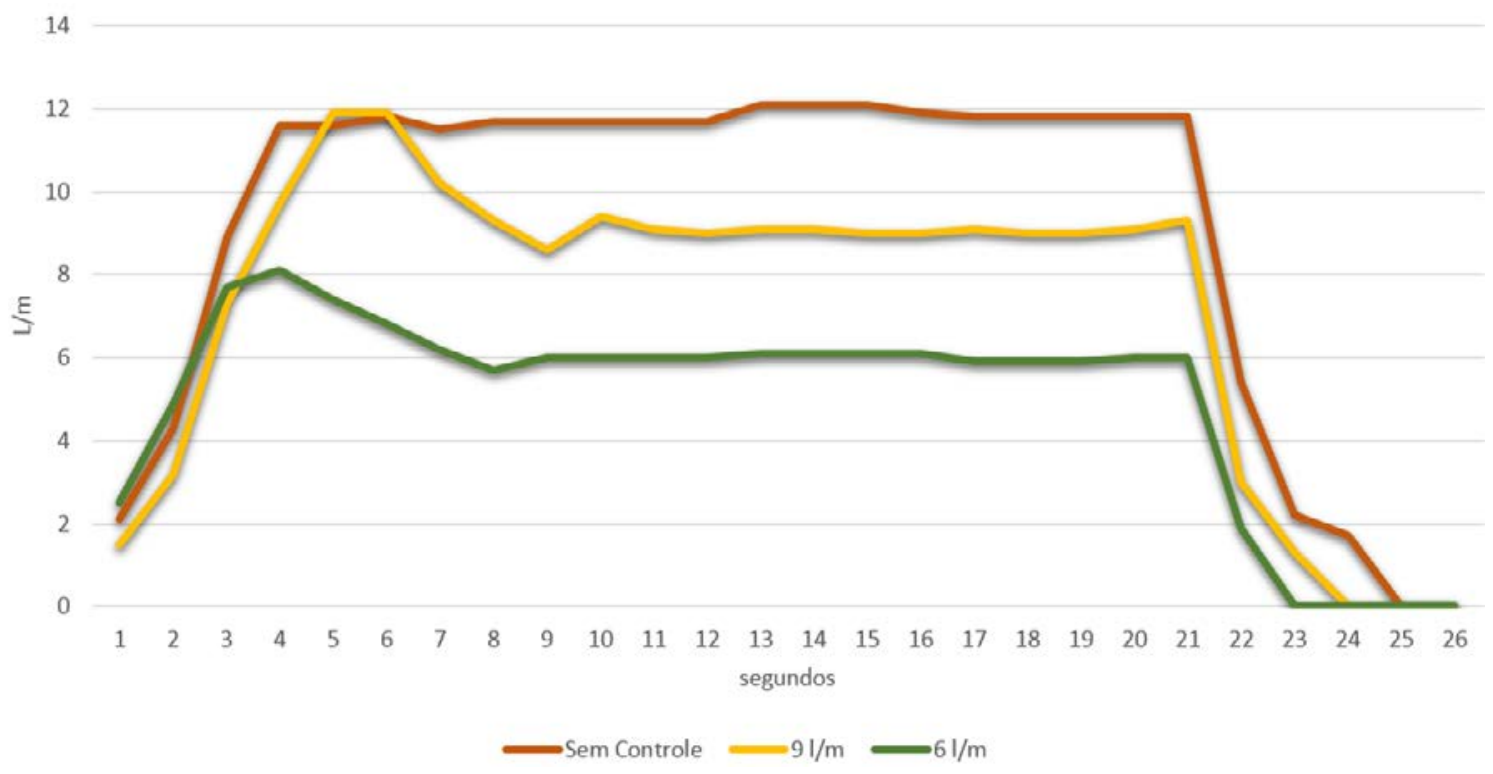

Figura 5. Experimentos de redução do controle de fluxo de água

no protótipo, por meio da detecção facial, para que posteriormente seja possível monitorálo e decisões sejam tomadas a respeito do possível uso excessivo da água na residência.

Desenvolveu-se o protótipo do controlador de fluxo com o objetivo de analisar a viabilidade da construção do sistema que permitisse controlar, de forma dinâmica, o fluxo de água. No resultado final do controlador, verificou-se que o protótipo apresentou folgas que não permitiu a relação direta entre a vazão e a posição do servo motor ( 0 a 180 graus), no entanto, o software desenvolvido para sanar o problema permitiu que o controlador atuasse de acordo com a proposta.

Os experimentos com o controlador de fluxo mostraram que é possível que o usuário tenha total controle sobre o fluxo de água em um dispositivo hídrico qualquer. Nos experimentos, definiu-se a vazão de 6 litros/minuto como o valor mínimo aceitável para que uma tarefa doméstica não seja prejudicada. Com esse valor, obteve-se uma economia de 50\% em uma torneira que tinha como média de vazão 12 litros/minuto.

Dentre os trabalhos futuros está a integração desse sistema com o controle térmico da temperatura de um recurso, como temperatura da água ou do ambiente, permitindo que utilizadores diferentes tenham diferentes perfis de utilização. Um exemplo dessa personalização é o ajuste da temperatura do chuveiro e torneiras de acordo com o utilizador, bem como o ajuste da temperatura do ar condicionado no local onde a pessoa se encontra.

\section{Referências}

Ahonen, T., Hadid, A., and Pietikainen, M. (2006). Face description with local binary patterns: Application to face recognition. Pattern Analysis and Machine Intelligence, IEEE Transactions on, 28(12):2037-2041.

Atmel (2014). Atmell 8-bit microcontroller with 4/8/16/32 kbytes in-system programable flash datasheet. 
Bolzani, C. A. M. (2004). Residências inteligentes. Editora Livraria da Física.

Bradski, G. and Kaehler, A. (2008). Learning OpenCV: Computer vision with the OpenCV library. "O'Reilly Media, Inc.".

Chan, C.-H., Kittler, J., and Messer, K. (2007). Multi-scale local binary pattern histograms for face recognition. Springer.

Diniz, F. A., Neto, F. M. M., Júnior, F. d. C. L., and Fontes, L. M. O. (2013). Redface: um sistema de reconhecimento facial baseado em técnicas de análise de componentes principais e autofaces. Revista Brasileira de Computação Aplicada, 5(1):42-54.

do Amaral, V., Giraldi, G. A., and Thomaz, C. E. Lbp estatıstico aplicado ao reconhecimento de expressoes faciais.

FAO (2011). The state of the wolrd's land and water resources for food and agriculture (SOLAW) - Managing systems at risk. Rome and Earthscan, London.

Morais, R. (2015). Morador do df usa 67\% mais água ao dia do que o indicado pela ONU.

Ojala, T., Pietikäinen, M., and Harwood, D. (1996). A comparative study of texture measures with classification based on featured distributions. Pattern recognition, 29(1):5159.

Rizzoli, A. C. M. G. D. P. A. C. A. (2015). Benefits and challenges of using smart meters for advancing residential water demand modeling and management: A review. Environmental Modelling and Software, 72:198214:62.

SNSA (2014). Diagnóstico dos Serviços de Água e Esgoto - 2013. SNSA/MCIDADES, Brasília.

Sohraby, K., Minoli, D., and Znati, T. (2007). Wireless sensor networks: technology, protocols, and applications. John Wiley \& Sons.

Suprotim Sinha Majumdar, M. P. and Alamelu, J. (2015). Energy efficient wireless sensor network for polyhouse monitoring. European Journal of Advances in Engineering and Technology, (2.6):77-82.

Viola, P. and Jones, M. J. (2004). Robust real-time face detection. International journal of computer vision, 57(2):137-154.

WWAP (2015). The United Nations World Water Development Report 2015 - Water for a sustainable world. UNESCO, Paris.

Zhang, B., Gao, Y., Zhao, S., and Liu, J. (2010). Local derivative pattern versus local binary pattern: face recognition with high-order local pattern descriptor. Image Processing, IEEE Transactions on, 19(2):533-544.

Zhao, W., Chellappa, R., Phillips, P. J., and Rosenfeld, A. (2003). Face recognition: A literature survey. Acm Computing Surveys (CSUR), 35(4):399-458. 